\title{
Examining Correlation Between the Genre of Music Present \& Increased Academic Performance Through Beta Wave Measurement
}

\author{
Daksha Magesh ${ }^{1}$ and Daniel Yan ${ }^{1}$
}

${ }^{1}$ Tesla STEM High School, Redmond, WA, USA

\begin{abstract}
$\underline{\text { ABSTRACT }}$
In a world saturated with media at every turn - especially music, and many different genres of music for everyone to enjoy, the critical question is often asked: does music positively impact the work produced whilst listening to it? This experiment seeks to answer that question using a 3D printed EEG to measure beta waves in the brain, thus providing more concrete results. It was hypothesized that listening to a genre of music such as classical would boost performance on a math exam. This experiment explores the correlation between the presence of music whilst taking a math exam and the score on said exam. In the experiment, each participant was first given a math exam to complete with no music whilst undergoing an EEG examination to establish a baseline, then were given a different form of the exam to complete whilst listening to a specific genre of music that was randomly assigned prior. Afterwards, we determined that slight improvement was observed with an average increase in score of 0.45 points, however with an r-squared value of only 0.091. The EEG analysis did provide an r-squared value of 0.370 when experimental error was removed, giving a moderately strong relationship that music does not have an impact on beta wave amplitude. We concluded that although music increased math scores slightly, it was not statistically significant using an alpha level of 0.05 . However, since almost $20 \%$ of the trials had error resulting in unreliable data, further refinement/re-testing of the methodology must be conducted.
\end{abstract}

\section{Rationale}

The brain is defined as "an organ of soft nervous tissue contained in the skull of vertebrates." Contrary to its rather lackluster description, it is the key component to our survival, evolving constantly to keep up with our ever-changing society. The brain is composed of several elements which control the different parts of the human body, from something as tangible as arm movement to one's inner psyche. However, one thing that our brain is constantly doing is learning, and it differs from person to person in the way that it does so. Students in high schools everywhere use different methods to help them focus, from taking breaks every hour to listening to different types of music. The effectiveness of music has been proven by the Mozart Effect, defined as the "short term improvement on spatial temporal reasoning" (Cabanac et al. 1) wherein noticeable improvements on stress and grades can be observed. The Mozart Effect is thought to be a result of the brain, overcoming cognitive dissonance, and is regularly observed prominently in musicians. In a study done on 184 students of a secondary school in Quebec, comparing taking or not taking music class in secondary school, strong evidence of those enrolled and actively involved with music doing better in school was observed.

However, while some students view music as a comforting white noise, others complain that it is distracting, and does not help them at all. Teachers have taken to making playlists of music to play during work time, often comprised of student suggestions. Some even use a new device called binaural beats, which is rhythmic audio that is said to sync to your brainwaves and help students focus. Through this experiment, we intend to use an EEG to measure beta wave amplitude while playing different genres on music and measure its effect on academic performance through 
a basic math test. Beta waves, intended to measure alertness, concentration, and focus will give us quantitative data on if music affects academic performance. Our experiment primarily focuses on measuring academic performance in mathematical studies, because it is fairly novel and has not been tested on high school students. Using this experiment, we hope to create a positive effect on academic performance, and provide a classroom setting which is productive and focused.

\section{Literature Reviews}

Article 1: Huang, Rong-Hwa, and Yi-Nuo Shih. "Effects of Background Music on Concentration of Workers.” Work (Reading, Mass.), U.S. National Library of Medicine, 2011

Background music is a daily part of people's lives, used in the classroom setting or in the workplace to provide entertainment or to provide a white noise that will help focus. However, how background music affects the workplace and effectiveness has become a key topic of study. Several people have reported that it helps concentration, while others complain that it is nothing but a distraction. Background music is popular because of its somewhat therapeutic effect. Many investigations where findings such as the ability of music to influence heart rate and provide a unique stimulus have been conducted.

The purpose of this study was to analyze how different types of background music and participant's preference for them can affect attention through a Randomized Controlled Trial. The sample size was 89 people, most of them between the ages of 19 and 28 . The hypotheses predicted that (when compared to no background music) that one of two things could occur: 1), the background music would affect the attention of participants, 2) the preference that participants had for the music would affect their attention. "Chu's Attention Test" is an attention evaluation test frequently used in China and was used to quantify the attention of the participants. The types of background music that were played were pop music, classical music, and traditional Chinese music. The eighty-nine participants were randomly assigned to 4 groups, one being the control, and the rest for the different types of music. The results showed that participants listening to background music tend to score lower than those without, with the score averaging 104.9 with no background music but progressively getting worse, with pop music's average score being 98.2. In terms of the participant's liking for the music, participants listening to the music were asked to give it a score from 1-5 (1 being dislike). Those who assigned more extreme scores (closer to 1 or 5) tended to score lower on the attention test. The study concluded that while background music influenced attention test scores, the results were not statistically significant.

Article \#2: Antony, M. \& Priya, V.V. \& Gayathri, R.. (2018). Effect of music on academic performance of college students. Drug Invention Today. 10. 2093-2096.

Music has surrounded children for a long time, occupying an important role in academic achievement. Research has shown that there is a connection between awareness of sound and literacy skills, and it has been proposed that academic music programs can help children develop multi-sensory awareness. Educators maintain the strong belief that not only does music allow students to healthily express themselves, but also reaches students who are not as proficient in traditional academic subjects. Certain cross-sectional studies have shown that detection of pitch of patterns by participants can predict future performance on phonological and reading tests.

The purpose of the study was to determine the influence of music on the academic performance of college students, of which 80 random students were selected. They took a survey which asked questions about their opinions on the effect of music on academics and whether or not they believed that it had a positive or negative effect on their own grades. Questions on their interest in music and how they thought music would increase efficiency were specifically included. The results of the survey showed that the students who listened to music also reported higher academic 
performance. $40.5 \%$ of students listened to music sometimes, 64.3 of the students reported that their efficiency increased. The study concluded that while music is not the single solution to improving academic performance, it has the potential to advance academic achievement amongst students.

Article 3: Kesan, C., Özkalkan, Z., İriç, H., \& Kaya, D. (2012). The Effect of Music on the Test Scores of the Students in Limits and Derivatives Subject in the Mathematics Exams Done With Music.

Math and music have an interconnected relationship, and this has been investigated by many mathematicians and philosophers alike. One of the most famous studies conducted on this topic was known as the Mozart effect. Math test anxiety is also a huge issue, with many students becoming nervous due to lack of positive encouragement from the teacher. It has been shown through studies that even math games that are played with music have a positive effect on the class's achievement and student attitudes. It has been concluded from several studies that music therapy might be beneficial in anger management and other psychological issues.

The purpose of the study was to analyze the effect of music in exams on derivatives and limits completed by students on the test scores. Patterns of gender and types of music are also to be noted. The sample size of participants were 98 freshmen in Izmir Dokuz Eylul University, Buca Faculty of Education, and the Department of Elementary Mathematics Teaching in 2010-2011 fall semester. They were divided into 10 homogenous groups, and told 5 different types of music, then asked to rank the types of music to their preference. The types of music used were Nature music, soft rock, pop music, and classical music. Then, the students took the exam with and without music for a measured time period. The reliability coefficient calculated by a SPSS computer statistical program was .83. In the Limits topic, music "increased" the exam scores of male and female students, but the scores of those listening to classical music (as their first preference) were reduced. In the Derivatives topic, music "increased" the exam scores of both genders, but the scores of those taking the exam with nature or pop music (as their first preference) were reduced as well.

Article 4: Arnaud Cabanac, et al. (2013, August 21). Music and academic performance. - PubMed - NCBI. Retrieved from https://www.ncbi.nlm.nih.gov/pubmed/23973386

The Mozart Effect, as described by Cabanac et al., is a "short term improvement on 'spatial-temporal reasoning"” (Cabanac 1), wherein noticeable improvements on stress and grades can be observed. The Mozart Effect is thought to be a result of the brain, overcoming cognitive dissonance, and is regularly observed much more prominently in musicians. In a study done on 184 students of a secondary school, strong evidence of those enrolled and actively involved with music doing better in school was observed.

Among those involved in the study, in the years 2011-2012 third, fourth, and fifth year music students had an average of $2 \%$ higher overall grades than non-musicians had. For third years, the net score increase in Science classes was statistically significant, with a p-value od $<0.01$, fourth-year scores had statistical significance in sports, science, environmental science, and math with p-values of $<0.01,0.01,0.02$, and $<0.00$ respectively. In fifth year, students, math, history and socioeconomics had statistical significance with p-values of 0.03 each.

\section{Research Question}

Is there a correlation between mathematical academic proficiency and the presence of certain types of music through an examination of test scores $\&$ changes in beta wave amplitude? 


\section{Hypothesis}

Music will positively correlate with an increase in beta wave frequency, as it releases dopamine and adrenaline, causing a student to be more awake and alert. This will keep them focused and they will thus score higher on the math test scores. There might also be a correlation between the preference of music and test scores, as several other studies (see Bibliography) have noted that an extreme preference shows a correlation with worse outcomes on the process used to measure focus.

\section{Variables}

1. Mathematical academic proficiency: the change in overall math test scores across each test, where each test contains three (3) questions, with two (2) problems being worth 3 points, and one (1) question being worth 4 points, thus giving a total of ten points per test No calculator is allowed on the entire test. Grading shall be according to the standardized grading scale (see "Grading Scale"). Math tests are standardized to a maximum of 10 minutes in length but finishing sooner is allowed. The questions on the control test will differ when compared to the experimental test in questions, but not difficulty.

Topics that were used on the math test included only the following:

a. The following types of functions, all able to be solved without a calculator:

i. Polynomials to a maximum of a $4^{\text {th }}$ degree

ii. Rational functions

iii. Exponential and logarithmic functions

b. Systems of equations including only polynomial functions with at most 2 variables

c. A word problem involving any of the functions listed above

2. Presence of Music: a sample of music (see "Music Used") played at $70 \%$ volume over computer speakers for the duration of the examination.

\section{Participants}

For our participants, we chose to focus on high school students (aged: 14-18) across several schools located in the surrounding areas of Tesla STEM High School, including Lake Washington School District, Issaquah School District, Riverside School District, Bellevue School District.

\section{Recruitment}

For this experiment, we set up polls and notices on our social medias as well as sent out an email with a survey to students asking if they were interested in participating in our project. On the survey, there is an option that will allow them to show interest in participating in a trial, which allowed them to be contacted by us via email to further explain the experiment, as well as be provided with the necessary paperwork in order to participate in the study. At ANY TIME IN THE EXPERIMENT, the participant IS ALLOWED TO LEAVE IF THEY WISH.

\section{Materials list}

- OpenBCI wires to connect electrodes to Cyton board (on headset): https://shop.openbci.com/collec-

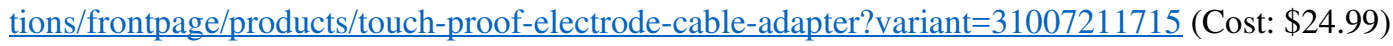

- Stopwatch (online)

- Researching space (rented a conference room at the King County Redmond Library) 
- EEG Headset - provided by Ms. Allender (AP Psychology and Forensics educator at Tesla STEM High School)- transmits data via Wi-Fi

- OpenBCI program, downloaded from the OpenBCI website to record data

- Personal laptop to run the program

- Alcohol swabs (to clean the electrode cleats)

- Electrode Cream (to increase connection and provide better data collection)

- Electrode Saline wash (to increase connection and provide better data collection, clean electrodes

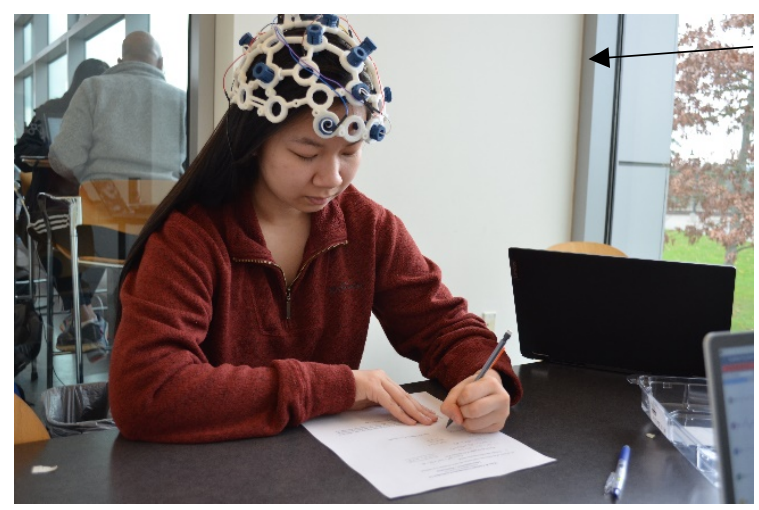

Figure 1: Participant taking the math test

whilst undergoing EEG examination

\section{Music Used}

Each genre of music tested has a sample of enough music to meet a length of 10 total minutes. These will cycle through for each participant based on which experimental group they are in.

\section{Classical:}

- "Jupiter" - Wolfgang Amadeus Mozart Symphony No. 41 in C Major

- "Fur Elise" - Ludwig Von Beethoven

- "Pavane" - Gabriel Faure

Pop:

- "Senorita" - Camila Cabello and Shawn Mendez

- "Girls Like You" - Maroon 5

- "Shape of You" - Ed Sheeran

\section{Binaural Beats:}

- "Focus music, Binaural Beats Concentration Music for Studying, Super Intelligence" [www.youtube.com/watch?v=UOeLmyJkQBc]

\section{Sampling Procedure}

1. Through a voluntary participation survey (sample attached, see "Survey"), collect responses and interest in participation for experiment.

a. If interested, have individual and a parent/guardian of individual sign the informed consent form.

2. Using responses of people interested in experiment participation, assign each possible participant with a number, beginning with 01 and increasing by 1 for each volunteer. 
3. From the set of volunteer participants, using a random number generator to select their numbers, select a set of 10 volunteers to serve as the $1^{\text {st }}$ testing group.

4. Repeat step 3 for the other 2 testing groups, skipping any repeat draws of participants

5. Each participant will only complete a trial for one type of music along with controls.

\section{Experimental Procedure}

1. Bring the participant into the test area

a. Reassure them about the test, calm them down

b. Note their participant number, age/grade, and email address

c. Confirm that they do not have any scalp issues that may produce a risk of infection (severe dandruff, lice)

d. Record their number from the sampling procedure (no personal details except for age shall be recorded that are tied to the results) - this number shall act as an identifier for the participant, without revealing said participant's identity.

2. Briefly explain the experiment and the purpose in order to reduce confusion and get confirmation of final consent, as well as what an EEG does in the experiment

a. The EEG will be collecting brainwaves for us to analyze, particularly beta waves, which measure the degree of alertness and focus that the participant is feeling

3. Provide them with a seat and carefully affix the brain cap of the EEG to their head, adjusting the electrodes to comfortably rest against the participant's scalp.

4. Open the OpenBCI software and perform setup as needed:

a. Move the switch on the side of the cyton board on the back of the EEG to the 'Bluetooth setting'

b. On the software setup, connect the headset to the program.

c. The electrodes will start picking up data: each color corresponds to a different area of the brain which the electrode is recording brain waves from. 


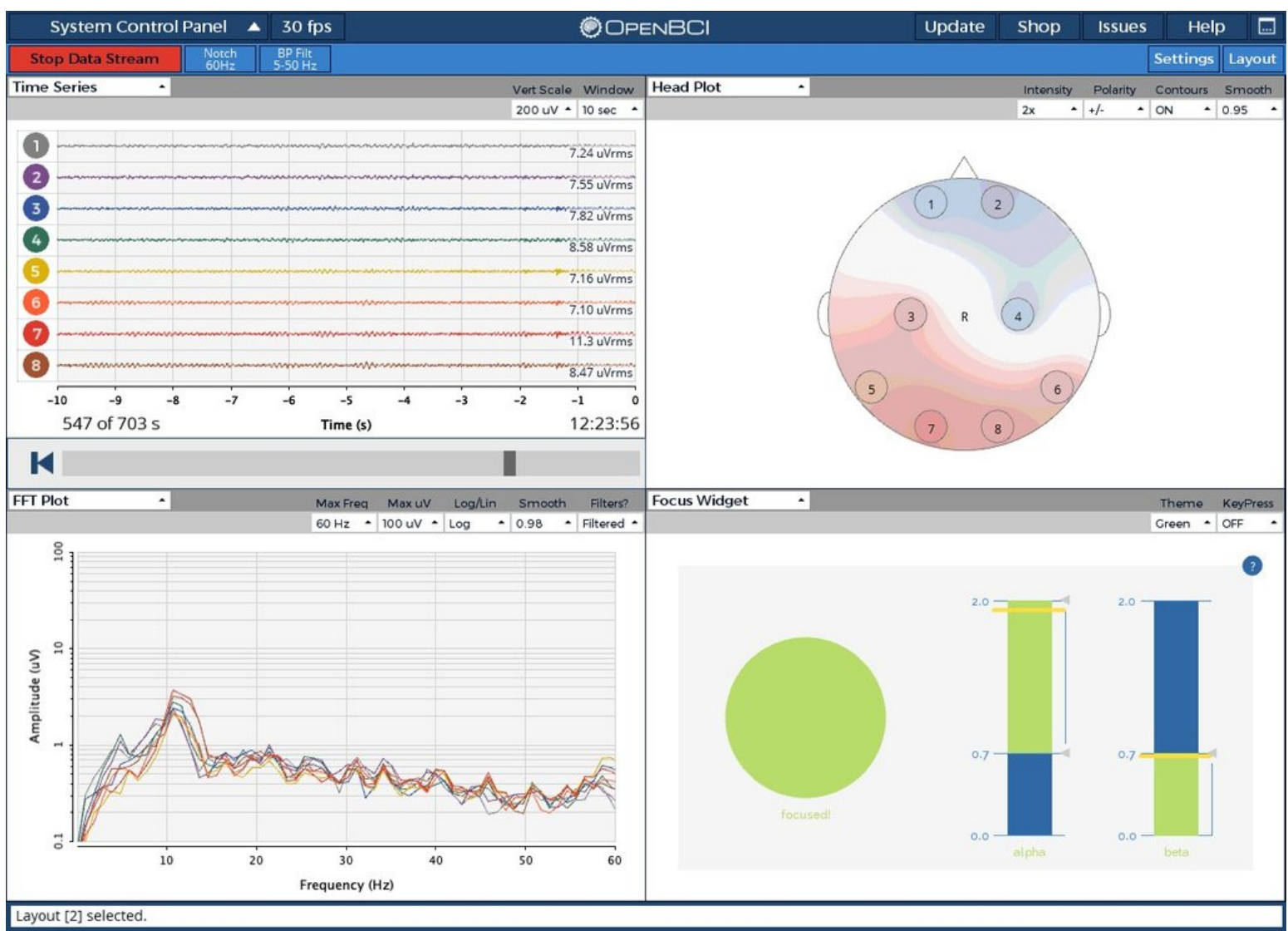

Figure 2: Example of OpenBCI program user interface

Run the following controlled variable experiment for 10 minutes:

d. Only taking the math test: (see closer description under "Variables")

- Record the subject's brainwave activity on the OpenBCI program using a screen recorder app while they are taking the math test and not listening to any music. This math test will be different from the test they will take during the actual experiment.

5. Provide the actual experimental test (see "Variables"), face down. Do not allow the participant to look at test before time starts.

6. Start playing the music on a speaker at $70 \%$.

7. Simultaneously say "Start" to the participant and start a stopwatch for 10 minutes as this is the limit for how long the participant has to complete all questions on the given math test. 




Figure 3. Researcher Daniel Yan and participant begin the EEG examination at the library

8. Begin recording the subject's brainwave activity on the OpenBCI program using a screen recorder app.

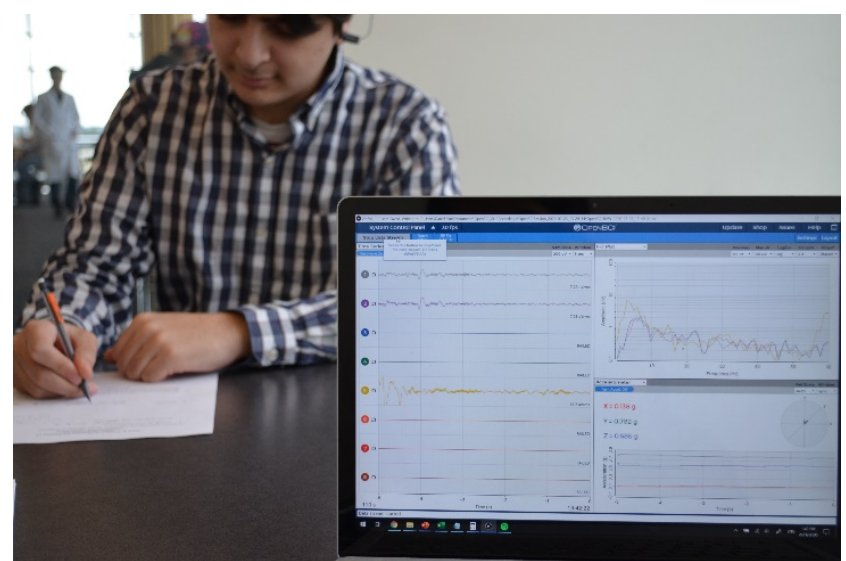

Figure 4. Researcher Daksha Magesh takes a photo of a participant's EEG readings as he takes the math examination.

9. After the participant has completed the test or the stopwatch reaches 10 minutes, end the test.

a. Collect the test and writing utensil from them.

b. Save the recorded data under a folder with the participant's profile number to avoid confusion.

10. Carefully remove the electrodes and brain cap from the participant's head, being sure not to cause the participant any discomfort.

11. Use an alcohol swab to gently clean the electrodes by swiping them across the small pinpoints on the tip to prevent cross contamination and reduce risk of infection.

12. Ask the participant to rate their enjoyment of the music on a scale of 1-5 and write this down.

13. Thank the participant for participating in the correlational study and explain that you will contact them later for their feedback and their experience.

14. Repeat steps 1-12 for each member of the $1^{\text {st }}$ experimental group. Record all data.

15. Repeat steps 1-12 for the next 2 experimental groups.

\section{Risk and Safety}

a) Cross contamination: The primary risk is that the electrodes are going to be in contact with human skin (scalp), and thus there is a risk of cross contamination/infection. We will minimize this risk by gently cleaning the electrodes with alcohol swabs/wipes. In addition, the patient will also be informed that if they have a skin 
condition that is contagious (EX: lice), they will be prohibited from participating in the study to ensure safety of other participants and prevention of cross contamination.

b) Mild Irritation: The electrodes used to collect data have slightly sharp cleats which rest on the scalp, which may cause a feeling of itchiness or mild irritation. We will minimize this risk by asking the participant if they are uncomfortable while setting up the EEG headset, as well as adjusting the electrodes so they are not digging into the scalp. In addition, we also have electrode cream and saline washes to increase connection and better data collection without having to press the cleats into the participant's scalp.

c) Interaction with Participants: Our experiment uses a math exam, and an EEG analysis will be minimally invasive, with the EEG being the only physical object that is in contact with the participant. Time required for this experiment is fairly short, requiring only a $30-45$-minute commitment at maximum that will be flexible, in order to be minimally obtrusive in the participant's day, if at all. This study will benefit the same population that it has studied, in being able to better scientific advice for or against a musical auditory stimulus while studying or taking academic exams.

d) Protection of Privacy: Critical identifiable information of participants will not be collected except for age and email address/phone number to contact them and arrange time to conduct experiment. Only age will be attached to data while email and/or phone number will be immediately removed once experiment on participant is completed. All data will be confidential, and test scores will be kept anonymous. Human Consent forms will immediately be shredded after the experiment is completed. Other participants will not have any access to the data, and all the data will be stored by those conducting the experiment. Only researchers Daksha Magesh and Daniel Yan have the authority to access the data, and the data will be kept after the experiment, but only for validity and to advance research in the topic we are studying.

e) Informed Consent Process: Participants will be distributed a copy of the informed consent form, as well as hear all the information on the informed consent form before the test begins. All testing is voluntary, and at any point if the participant feels uncomfortable or not willing to continue, they may stop at any point. They will be asked to only complete two, 10-minute long tests, as well as sit in a room for 2 sessions of 1 minute and 30 seconds a piece - music may or may not play at any of the times they are in the room. All other data is collected via EEG which is recorded during the 10- and 1.5-minute intervals. EEG participation especially, is voluntary.

\section{Data Collection and Analysis}

\section{Data Collected}

- EEG amplitude for doing nothing, listening to music, taking a test, and listening to music while taking a test.

- Screen recordings of entire EEG tests for further analysis

- $\quad$ Test scores with and without music

- NOTE: Each participant will only test one condition

[ $\Delta$ Score] is calculated by: [math score with music] - [initial score]

[ $\triangle \mathrm{EEG}$ Amplitude] is calculated by: : [music EEG amplitude] - [control EEG amplitude]

After all data is collected and analyzed, (Initial Score, Music Score) was plotted for each participant on a coordinate grid, with the 3 experimental groups each having their own graph. Then a best fit line was found using linear regression. From this line, an $\mathrm{R}$ value as well as $\mathrm{R}^{\wedge} 2$ can be found. The change in beta wave frequency for each type of music is also plotted, so we can analyze the difference of $\mathrm{Hz}$ per participant. 


\section{Grading Scale}

The 3-point questions shall be scored according to the following:

- 0 points shall be awarded if:

- The problem is not attempted, and the space is left blank

- The work \& problem is incomplete

- The work is finished, but no clear answer is given and labeled

- 1 point shall be awarded if:

- Work and answer are given and clearly labeled, but the answer is off by more than: [actual answer $] \pm(2 *$ [actual answer $])$

- Answer is given, but no work is shown (regardless of correctness)

○ 2 points shall be awarded:

- Work and answer are given and clearly labeled, and the answer is off within the margin of:

[actual answer $] \pm(2 *$ [actual answer $])$ to [actual answer $] \pm(0.25 *$ [actual answer] $)$

○ 2.5 points shall be awarded if:

- Work and answer are given and clearly labeled, and the answer is off by a factor less than:

- 3 points shall be awarded if:

$$
\text { [actual answer }] \pm(0.25 * \text { [actual answer }]
$$

- Work and answer are given and clearly labeled, and answer exactly matches answer key, not accounting for a \pm 0.05 error margin due to rounding the answer

- No other scores shall be awarded

The 4-point question shall be scored according to the following:

0 points shall be awarded if:

- The problem is not attempted, and the space is left blank

- The work \& problem is incomplete

- The work is finished, but no clear answer is given and labeled

- 1 point shall be awarded if:

- Work and answer are given and clearly labeled, but the answer is off by more than:

$$
\text { [actual answer }] \pm(2.5 * \text { [actual answer }])
$$

- Answer is given, but no work is shown (regardless of correctness)

○ 2 points shall be awarded if:

- Work and answer are deserving of a 2.5-point grade, but answer is without context or units

○ 2.5 points shall be awarded if:

- Work and answer are given and clearly labeled, and the answer is off within the margin of:

[actual answer $] \pm(2.5 *$ [actual answer $])$ to [actual answer $] \pm(0.5 *$ [actual answer $])$ and the answer is given in context of the problem with units included

○ 3 points shall be awarded if:

- Work and answer are deserving of a 3.5-point grade, but answer is without context or units

○ 3.5 points shall be awarded if:

- Work and answer are given and clearly labeled, and the answer is off by a factor less than: [actual answer $] \pm(0.5 *$ [actual answer $]$ and the answer is given in context of the problem with units included

○ 4 points shall be awarded if:

- Work and answer are given and clearly labeled, and answer exactly matches answer key, not accounting for a \pm 0.05 error margin due to rounding the answer, and the answer is given in context of the problem with units included 


\section{No other scores shall be awarded}

\section{Data Analysis and Results}

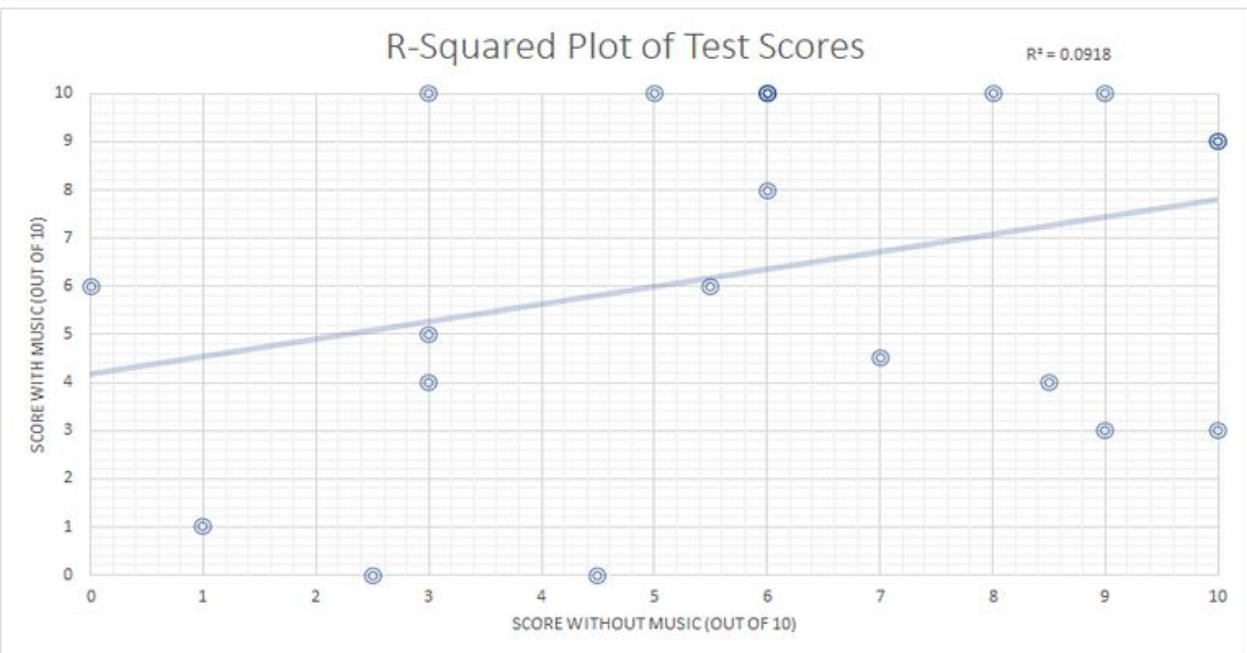

Figure 5. R squared plot of the math examination scores, with and without music

An r- squared value of 0.0918 was calculated from the data set when comparing test scores with and without the presence of music. An r-squared value of 0.0001 was calculated if artifacting EEG data trials was used, but an $\mathrm{r}$ squared value of 0.370 was calculated when artifacting EEG data was not considered.

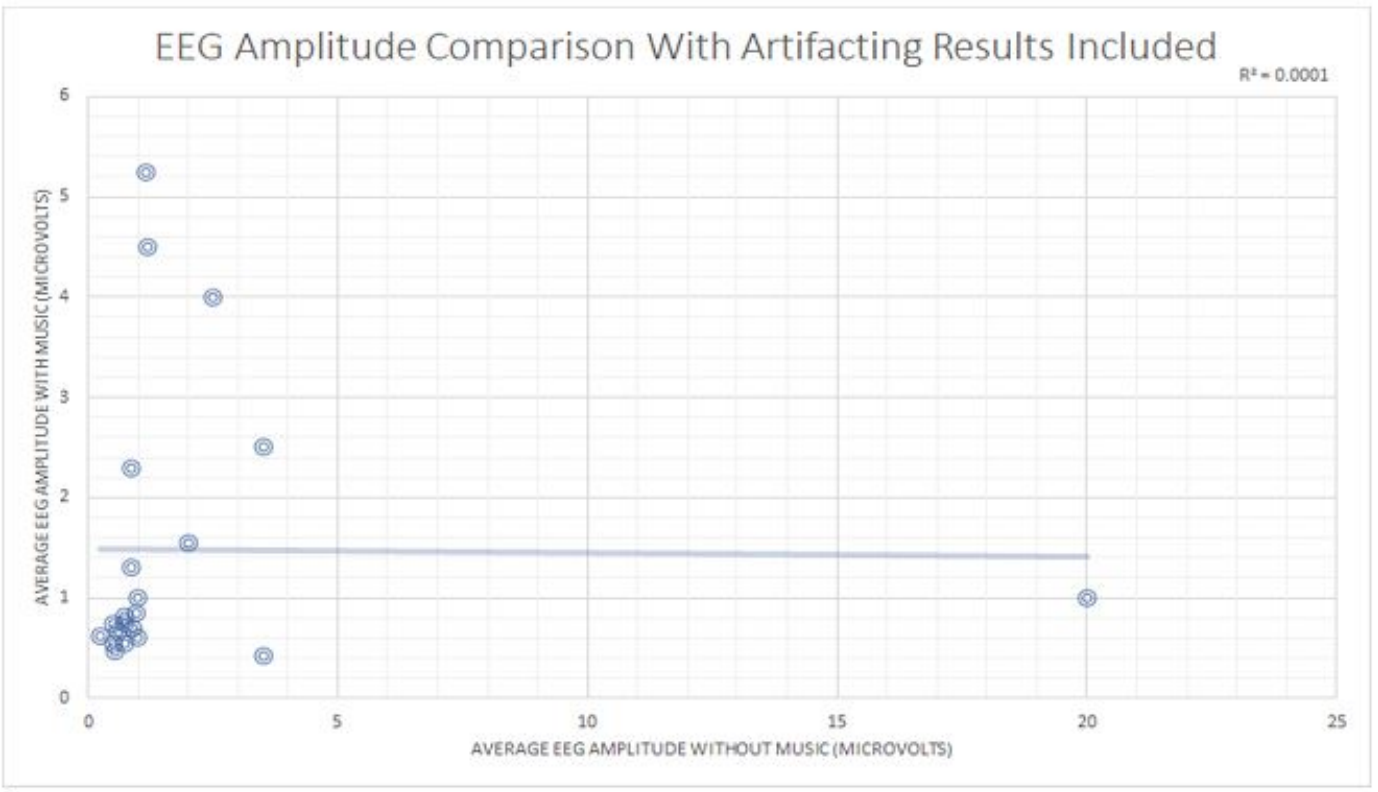




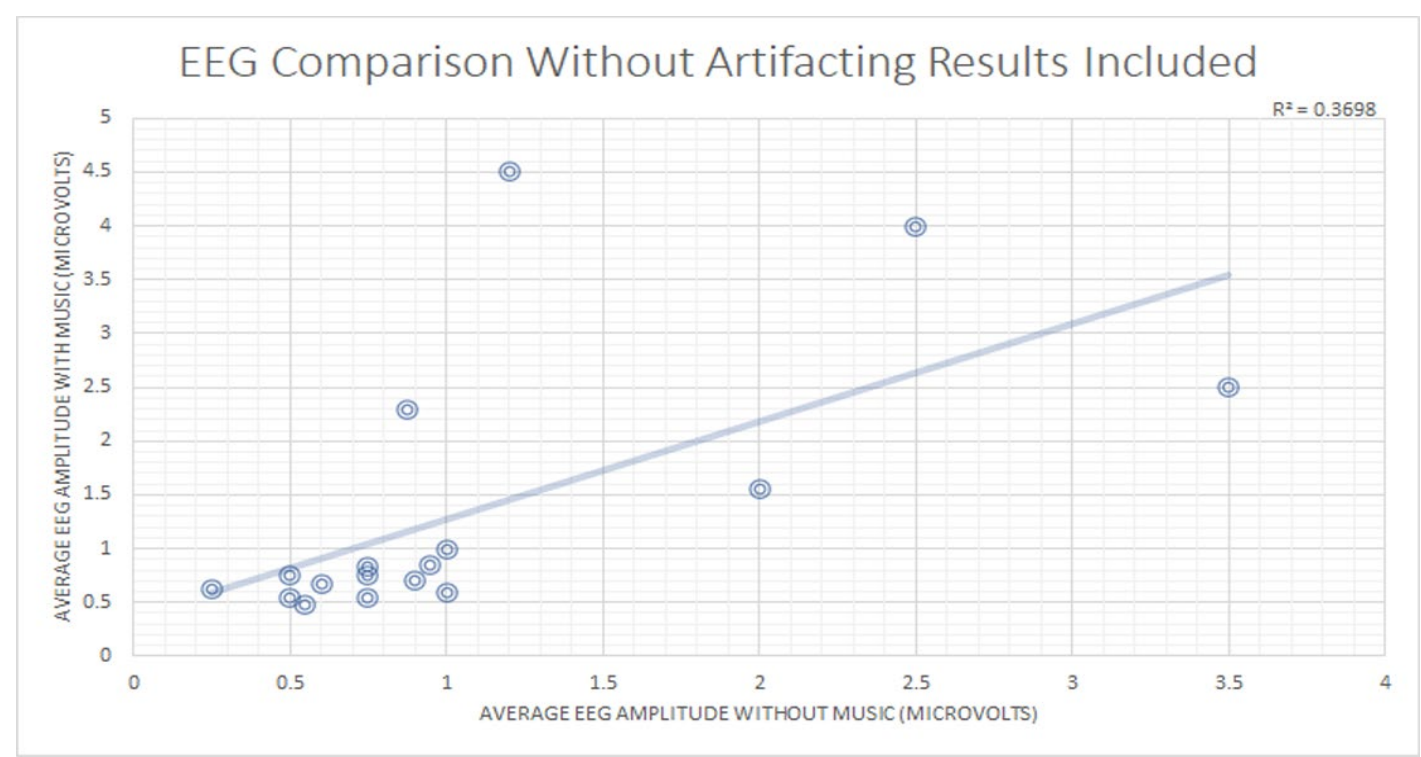




\begin{tabular}{|c|c|c|c|c|c|c|c|c|c|}
\hline $\begin{array}{c}\text { Subject } \\
\#\end{array}$ & $\begin{array}{c}\text { Type of } \\
\text { Music }\end{array}$ & $\begin{array}{c}\begin{array}{c}\text { Con- } \\
\text { trol } \\
\text { Test }\end{array} \\
\end{array}$ & $\begin{array}{c}\text { Music } \\
\text { Test }\end{array}$ & $\begin{array}{l}\text { Control } \\
\text { Score }\end{array}$ & $\begin{array}{l}\text { Music } \\
\text { Score }\end{array}$ & $\begin{array}{c}\text { Test } \\
\text { Change }\end{array}$ & $\begin{array}{c}\text { Control } \\
\text { EEG }\end{array}$ & $\begin{array}{c}\text { Music } \\
\text { EEG }\end{array}$ & $\begin{array}{c}\text { EEG } \\
\text { Change }\end{array}$ \\
\hline \#004 & Binaural & A & C & 3 & 5 & 2 & 0.75 & 0.75 & $\mathbf{0}$ \\
\hline \#009 & Pop & C & B & 6 & 8 & 2 & 1 & 1 & $\mathbf{0}$ \\
\hline \#012 & Classical & B & A & 10 & 3 & -7 & 0.5 & 0.75 & 0.25 \\
\hline \#015 & Pop & $\mathrm{C}$ & B & 3 & 4 & 1 & 0.6 & 0.675 & 0.075 \\
\hline \#016 & Classical & $\mathrm{C}$ & A & 1 & 1 & $\mathbf{0}$ & 0.5 & 0.55 & 0.05 \\
\hline \#017 & Binaural & A & C & 5.5 & 6 & 0.5 & 0.75 & 0.55 & -0.2 \\
\hline$\# 022$ & Binaural & $\mathrm{C}$ & $\mathbf{A}$ & 0 & 6 & 6 & 2 & 1.55 & -0.45 \\
\hline \#025 & Pop & A & C & 2.5 & 0 & -2.5 & 0.25 & 0.625 & 0.375 \\
\hline \#028 & Classical & B & $\mathbf{A}$ & 4.5 & 0 & -4.5 & 0.95 & 0.85 & -0.1 \\
\hline \#029 & Pop & A & C & 9 & 10 & 1 & 0.9 & 0.7 & -0.2 \\
\hline \#034 & Binaural & A & B & 6 & 10 & 4 & 2.5 & 4 & 1.5 \\
\hline \#036 & Pop & B & $\mathbf{A}$ & 10 & 9 & -1 & 0.55 & 0.475 & -0.075 \\
\hline \#039 & Binaural & $\mathrm{C}$ & B & 6 & 10 & 4 & 1 & 0.6 & -0.4 \\
\hline \#040 & Classical & B & $\mathbf{A}$ & 10 & 9 & -1 & 3.5 & 0.425 & -3.075 \\
\hline \#042 & Binaural & C & B & 6 & 10 & 4 & 0.75 & 0.825 & 0.075 \\
\hline \#046 & Pop & $\mathrm{C}$ & B & 3 & 10 & 7 & 20 & 1 & -19 \\
\hline$\# 047$ & Binaural & B & C & 7 & 4.5 & -2.5 & 1.2 & 4.5 & 3.3 \\
\hline$\# 048$ & Pop & A & B & 5 & 10 & 5 & 1.15 & 5.25 & 4.1 \\
\hline \#051 & Classical & C & A & 8 & 10 & 2 & 0.85 & 1.3 & 0.45 \\
\hline \#052 & Binaural & $\mathbf{A}$ & C & 8.5 & 4 & -4.5 & 0.875 & 2.3 & 1.425 \\
\hline \#054 & Classical & B & A & 9 & 3 & -6 & 3.5 & 2.5 & -1 \\
\hline
\end{tabular}


Table 1. Full Raw Data Table From Experiment. Orange cells indicate artifacting results from EEG exam and yellow represents near-artifacting results. When calculating statistical analysis, the trials with orange in the [EEG change] cell were not used in the second calculation.

- The participants scored an average of 5.86 points on the exams when there was no music playing in the room. When there was music playing, the participants scored an average of 6.31 points on the exams

- An average increase of 0.45 points was observed across the twenty-one participants when music was introduced versus when it was not.

- Music results: Binaural Beats had an average score increase of 1.69 points and an average EEG amplitude change of 0.66 microvolts, Classical music had an average score decrease of 2.75 points and an average EEG amplitude change of -0.2 microvolts, Pop music has an average increase of 1.76 points and an average EEG amplitude change of 0.035 microvolts

- Test Results: Test A had an average score of 5.28 points, Test B has an average score of 9.04 points, Test C had an average score of 4.46 points

- On average, there was a net change in EEG beta wave readings of 1.72 microvolts but there was actually an average decrease of 0.61 microvolts with music

Left: Data Collection during experimentation

Proper collected data

Artifacting data

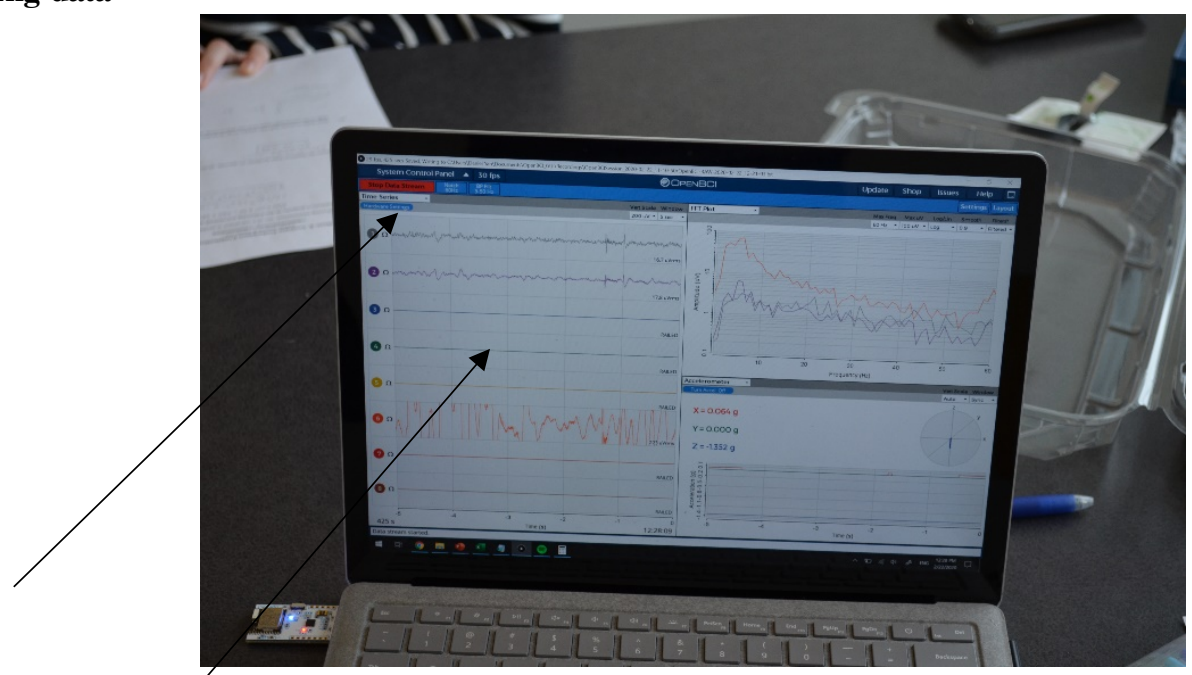

\section{Conclusion}

Through statistical analysis of all the data gathered, the significant data trend found was an r-squared value of 0.370, indicating a fairly strong relationship between EEG scores with and without music, the correlation coefficient was found to be 0.9 , showing that the EEG readings with and without music are usually fairly close to each other, indicating that music does not affect beta wave amplitude.

However, despite the fact that the data indicates certain trends such as pop music being the type of music that actually increased test scores the most, certain uncontrolled variables such as the damage done to the EEG by prior use resulted in almost $20 \%$ of our EEG data being unreliable. Due to this as well as the lack of reliability that the different tests showed, further refinement and re-testing of the methodology must be conducted to reinforce affirmation or refutation of the hypothesis.

The first step forward for this research would be a repeat of our methodology with more attention towards the extraneous variables not accounted for this time. We would seek to better standardize and revise the tests to make 
them more reliable. We would also seek to conduct the experiment with a more functional EEG with more electrodes intact in order to reduce/eliminate the artifacting present in the recordings of the EEG data.

\section{References}

Aguirre - Perez, D. M., Otero - Ojeda, G.A., Pliego-Rivero, F.B., \& Ferreira - Martinez, A. A. (2007, June). Relationship of working memory and EEG to academic performance: a study among high school students.

Arnaud Cabanac, et al. (2013, August 21). Music and academic performance. - PubMed - NCBI. Retrieved from https://www.ncbi.nlm.nih.gov/pubmed/23973386

Cheung, Mei-Chun \& Chan, Agnes \& Han, Yvonne \& Sze, Sophia. (2014). Brain Activity During Resting State in Relation to Academic Performance. Journal of Psychophysiology. 28. 47-53. 10.1027/0269-8803/a000107.

Hsu CC, et al. (2017, January 12). Analyze the beta waves of electroencephalogram signals from young musicians and non-musicians in major scale working memory task. - PubMed - NCBI. Retrieved from

https://www.ncbi.nlm.nih.gov/pubmed/28088577

Huang, Rong-Hwa, and Yi-Nuo Shih. "Effects of Background Music on Concentration of Workers." Work (Reading, Mass.), U.S. National Library of Medicine, 2011

Kesan, C., Özkalkan, Z., İriç, H., \& Kaya, D. (2012). THE EFFECT OF MUSIC ON THE TEST SCORES OF THE STUDENTS IN LIMITS AND DERIVATIVES SUBJECT IN THE MATHEMATICS EXAMS DONE WITH MUSIC. 